\title{
The PAH emission properties of an ensemble of UCHII regions in W49A.
}

\author{
David Stock $^{* a}$, Els Peeters ${ }^{a b}$ and William D.-Y. Choi ${ }^{a}$ \\ ${ }^{a}$ Department of Physics and Astronomy, University of Western Ontario, Canada \\ ${ }^{b}$ SETI Institute, Mountain View, CA, USA \\ E-mail: dstock4@uwo.ca
}

The galactic star-forming region W49A is considered to be the Milky Way analogue of extragalactic starburst environments. W49A contains an ensemble of ultra compact HII (UCHII) regions along with copious diffuse material and young stars. Spitzer/IRS mapping observations of a $3^{\prime} \times 2^{\prime}$ subsection of W49A have been obtained in the 5-14 $\mu \mathrm{m}$ range. These observations cover approximately $20 \%$ of W49A and encompass many of the previously detected UCHII regions along with diffuse structure, all of which display the characteristic mid infrared (MIR) PAH emission. The spectral properties of the emission at each pixel of the map have been analyzed, allowing the detailed comparison of the MIR emission of the different UCHII regions and surrounding material. The UCHII regions possess different properties in terms of their stellar populations (and hence the incident UV fields), ionization, extinction etc. resulting in different PAH emission characteristics. These results are compared to the characteristics of the diffuse PAH emission surrounding W49A, along with previous studies of PAHs in HII regions. Furthermore, we investigate the link between the PAH emission and the physical conditions of the HII regions (e.g. line ratio proxies for ionization). Finally, we show that the spatial structure of the various MIR emission components in these UCHII regions (e.g. the continuum emission, PAH bands and forbidden lines) can be simply modeled assuming emission from spherically symmetric shells. This model can recover the parameters of the emitting regions, e.g. the characteristic radii and thickness of the emitting shells. Model fits then show that the $8.6 \mu \mathrm{m}$ PAH emission originates closer to the exciting stars than the other PAH bands. In addition, for one of the UCHII regions, we find that the 6.2 and 7.7 $\mathrm{PAH}$ bands no longer correlate on the lines of sight near the center, an effect noted previously in only one other object, also an HII region. It is also shown that the continuum emission and the plateaus supporting the 5-10 $\mu \mathrm{m}$ PAH features are spatially disconnected in these objects.

The Life Cycle of Dust in the Universe: Observations, Theory, and Laboratory Experiments 18-22 November, 2013

Taipei, Taiwan

\footnotetext{
* Speaker.
} 

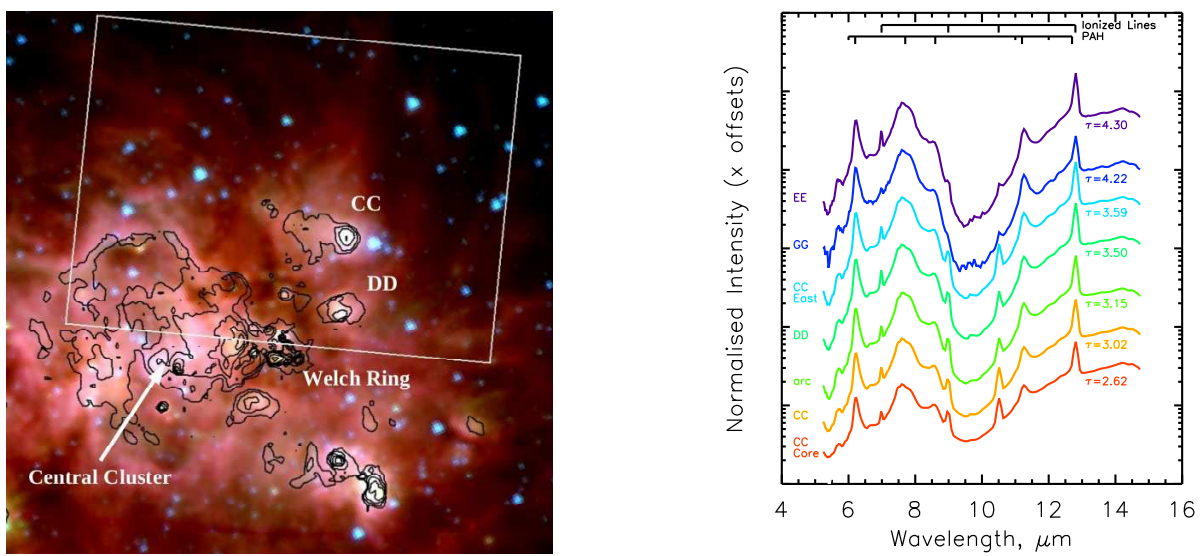

Figure 1: left: The field of view of the Spitzer/IRS spectral cube (white square) overlaid on a three color IRAC image ( $\mathrm{R}=[8.0], \mathrm{G}=[5.4], \mathrm{B}=[3.6])$ from the GLIMPSE survey [3] with north up and east to the right. Black contours represent the radio continuum observations of [5]. Some regions of interest have been labeled in white. The coordinates of the north eastern corner of the field of view are: $19 \mathrm{~h} 10 \mathrm{~m} 18 \mathrm{~s}+9^{\circ} 8^{\prime} 48^{\prime \prime}$. Right: The integrated spectra of the various HII regions in the W49A IRS cube field of view. Note the presence of the silicate absorption feature at $9.8 \mu \mathrm{m}$ in each spectrum, the optical depth of which is shown for each spectrum.

\section{Introduction}

The galactic star formation region W49A is frequently used as an analogue for extra-galactic star formation. In this work we investigate the links between H II regions, massive star formation complexes and extragalactic star formation in terms of the mid-infrared (MIR) polycyclic aromatic hydrocarbon $(\mathrm{PAH})$ emission bands. Throughout this work we have employed a large $3^{\prime} \times 2^{\prime}$ Spitzer/IRS-SL spectral map covering the 5-15 $\mu \mathrm{m}$ region at a spatial resolution of around 3.6". The field of view of these observations covers the northern section of W49A and is shown in Figure 1, left.

\section{Spectra and Extinction}

Galactic star forming regions are known to be afflicted with heavy extinction, in the MIR this manifests itself mainly in the silicate absorption feature at $9.8 \mu \mathrm{m}$. In Figure 1 (right) we show the IRS spectra of each of the ultra compact (UC) HII regions in W49A. Each shows a degree of silicate absorption consistent with previous line of sight measurements of $A_{K} \simeq 2.1$. In addition, each of the presented spectra shows a typical HII region MIR spectrum, with strong PAH emission from the associated PDR. In a previous work, we implemented a method of measuring this feature and dereddening MIR spectra [7]. Following [价, we extinction corrected each pixel of the IRS-SL cube using the MIR extinction law given by [2].

\section{Anomalous PAH Emission}

In general, the appearance of the PAH emission in terms of the peak positions and relative strengths of the bands follows that observed in previous objects well, with one exception: the shell 

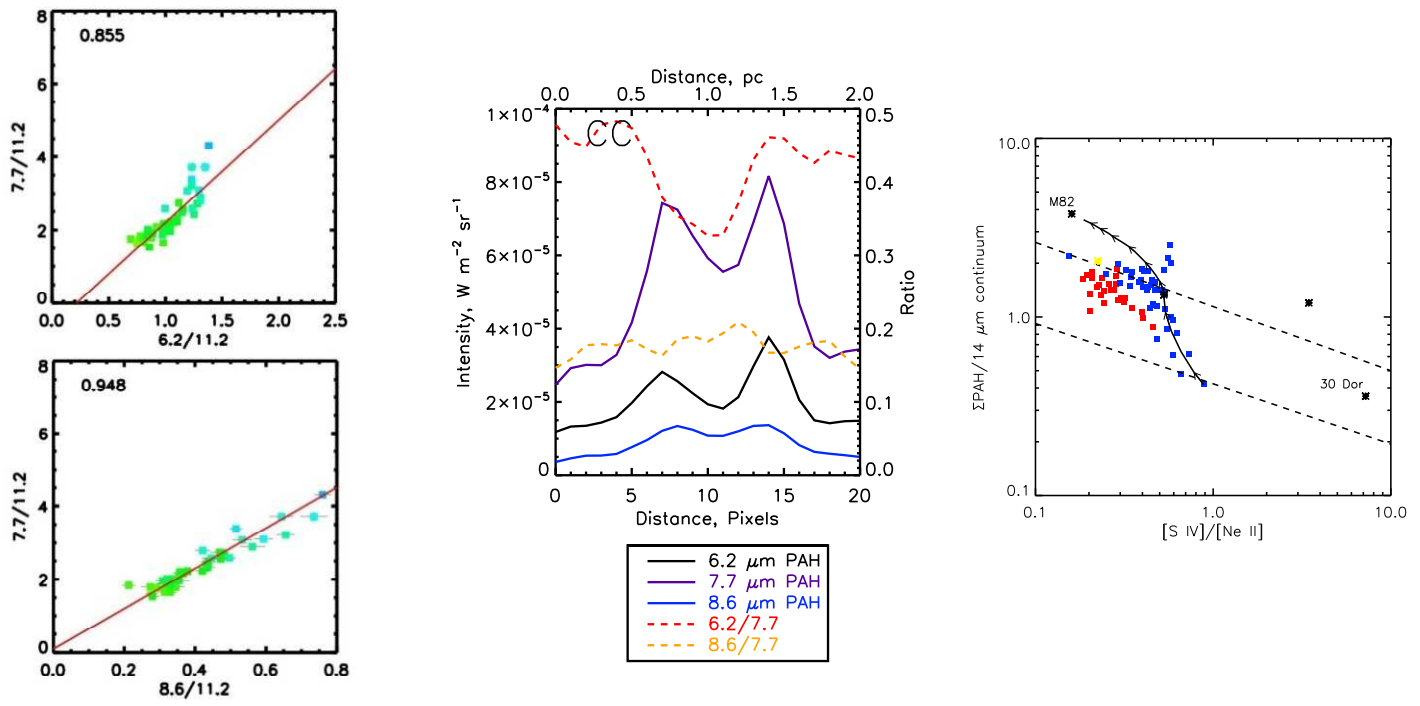

Figure 2: left: Correlation plots for the central region of UC HII region CC color coded by extinction $\left(\mathrm{A}_{K} \sim\right.$ $2-3$, blue - green). Note that for 6.2/11.2 vs 7.7/11.2 there is a 'knee' in the correlation at around 6.2/11.2 1.3, while for 8.6/11.2 vs 7.7/11.2 the expected linear relationship is recovered. Center: Normalized spatial emission profiles of UC HII region CC. The 6.2 PAH band does not follow the 7.7 and 8.6 bands for this object (e.g. very low ratio in center). Right: Relationship between [S IV]/[Ne II] and PAH emission/14 $\mu \mathrm{m}$ continuum for UCHII regions CC (blue) and DD (red).

type UC HII region CC. Normally the $6.2 \mu \mathrm{m}$ PAH band forms a tight linear correlation with the $7.7 \mu \mathrm{m}$ PAH band when both are normalized to the $11.2 \mu \mathrm{m}$ band. However, as we show in Figure 2 (left), the 6.2 band variation with 7.7 is not linear, rather it has a definite knee at 6.2/11.2 1.3. This effect has only been previously observed in one object prior to this study: the LMC star forming region N66 [8]. We further investigated this problem by inspecting the spatial emission profiles in cuts though CC. These cuts are shown in Figure 2 (center), and show this effect most strongly in the 6.2/7.7 band ratio, in which the central minimum ratio is markedly less than the normal range of $0.45-0.8$ (e.g. [四).

\section{W49A and Star Formation}

The MIR PAH emission is commonly used as a star formation indicator and has been calibrated against other measures of star formation [1]]. In extragalactic contexts though the star formation complexes are usually unresolved. What is the relationship between the appearance of the resolved star formation complex W49A and unresolved extragalactic star formation? To investigate this effect we consider the appearance of W49A in terms of the [S IV]/[Ne II] vs PAH emission/14 $\mu \mathrm{m}$ continuum relationship (see Figure 2, right), which has been shown to separate HII regions and starburst galaxies effectively [6]. The W49A points straddle the HII region and starburst regimes, as one might expect given the presence of HII regions and PDR material. Starburst galaxies are comprised mainly of regions similar to W49A, however the spatial resolution of observations for starburst galaxies is much lower: an effect we can mimic by increasing the beam size in the W49A observation. In order to track the effect of increasing beam size we have included a track in Fig- 
ure 2 (right) which considers larger spatial apertures centered on UC HII region CC and thus the progression in spectral appearance from HII region to starburst galaxy as more PDR material is included in the beam.

\section{Conclusions}

1. The appearance and correlations of the PAH bands in W49A is consistent overall with previous observations,

2. in the shell of UC HII region CC, the correlation between the 6.2 and 7.7 PAH bands breaks down in a way only observed before in one other object,

3. W49A suffers from considerable extinction,

4. W49A mimics the spectra of starburst galaxies when observed on scales larger than its UC HII regions. On smaller scales the UC HII regions themselves behave in the same way as other Galactic UC HII regions.

\section{Acknowledgments}

DJS and EP acknowledge support from an NSERC Discovery Grant and an NSERC Discovery Accelerator Grant. WDYC acknowledges support from an NSERC Undergraduate Student Research Award.

\section{References}

[1] Calzetti, D., et al., The Calibration of Mid-Infrared Star Formation Rate Indicators, 2007, ApJ, 666, 870

[2] Chiar, J. E., \& Tielens, A. G. G. M., Pixie Dust: The Silicate Features in the Diffuse Interstellar Medium, 2006, ApJ, 637, 774

[3] Churchwell, E., et al., The Spitzer/GLIMPSE Surveys: A New View of the Milky Way, 2009, PASP, 121,213

[4] Galliano, F., Madden, S. C., Tielens, A. G. G. M., Peeters, E., \& Jones, A. P. Variations of the Mid-IR Aromatic Features inside and among Galaxies, 2008, ApJ, 679, 310

[5] de Pree, C. G., Mehringer, D. M., \& Goss, W. M., Multifrequency, High-Resolution Radio Recombination Line Observations of the Massive Star-forming Region W49A, 1997, ApJ, 482, 307

[6] Lebouteiller, V., Bernard-Salas, J., Whelan, D. G., Brandl, B., Galliano, F., Charmandaris, V., Madden, S., \& Kunth, D., Influence of the Environment on Polycyclic Aromatic Hydrocarbon Emission in Star-forming Regions, 2011, ApJ, 728, 45

[7] Stock, D. J., Peeters, E., Tielens, A. G. G. M., Otaguro, J. N., \& Bik, A., Extinction and Polycyclic Aromatic Hydrocarbon Intensity Variations across the H II Region IRAS 12063-6259, 2013, ApJ, 771, 72

[8] Whelan, D. G., Lebouteiller, V., Galliano, F., Peeters, E., Bernard-Salas, J., Johnson, K. E., Indebetouw, R., \& Brandl, B. R., An In-depth View of the Mid-infrared Properties of Point Sources and the Diffuse ISM in the SMC Giant H II Region, N66, 2013, ApJ, 771, 16 